\title{
Investigation and Suppression of RF Leakage Power in Front End Wireless Devices
}

\author{
Kanchana Devi $\mathrm{A}^{\mathrm{a}, 1}$ and Bhuvaneswari $\mathrm{B}^{\mathrm{b}}$ \\ ${ }^{a} P G$ Scholar, Dept of ECE, Panimalar Engineering College, Chennai, India \\ ${ }^{\mathrm{b}}$ Professor, Dept of ECE, Panimalar Engineering College, Chennai, India
}

\begin{abstract}
In this modern Communication Wireless System, Frequency Division Duplex (FDD) is mostly used. Duplex is a device to separate Transmitter and Receiver signals. Transmitter or Power leakage causes from limited isolation performance of the duplexer. Various Techniques of Modulation using Orthogonal Frequency Division Multiplexing (OFDM) provided better solution to cancel this leakage. The OFDM provides high spectral efficiency, lower multi-path distortion and to eliminate inter symbol interference (ISI). Fast Fourier Transform implemented modulation and demodulation functions more efficiently. Using simulation result of the various parameters are analysed. In addition, Comparison of the table between Bit rate error value, Signal strength throughput, Power consumption and Mean square error values obtained in the OFDM systems.
\end{abstract}

Keywords. FDD, OFDM, ISI, BPSK, QPSK, 16-QAM, SNR, MSE and Power spectral density.

\section{Introduction}

In Communication System, the input streams are modulated in carrier frequency. The available spectrum bandwidth occupied fully by each of every symbol. This leads to inter-symbol-interference (ISI). OFDM gives sufficient robustness using larger data rates to radio channel [1]. In the world many researcher teams are working under OFDM system optimization. Block diagram of FDD transceiver is shown in figure 1. Since an FDD transceiver both transmit and receive at the same time, the function of duplex filter is connected the TX and RX to the same antenna [2]. The duplex TX/RX isolation is not infinite and it results in a leakage signal at the receiver input. Radio receiver circuit, the circuit between antenna and mixer called RF Front End Module. It consisted all the components in receiver processed the original signal before converted to lower intermediate frequency (IF). It is used to detect, band limiting and amplifying the received RF signals. RF amplifier needed to strength weak signals that are received increasing the amplitude of the RF signals that are weak[12].

\footnotetext{
${ }^{1}$ Kanchana Devi. A, PG Scholar, Dept of ECE, Panimalar Engineering College, Chennai, India, E-mail: akanchanadeviselvi@gmail.com.
} 


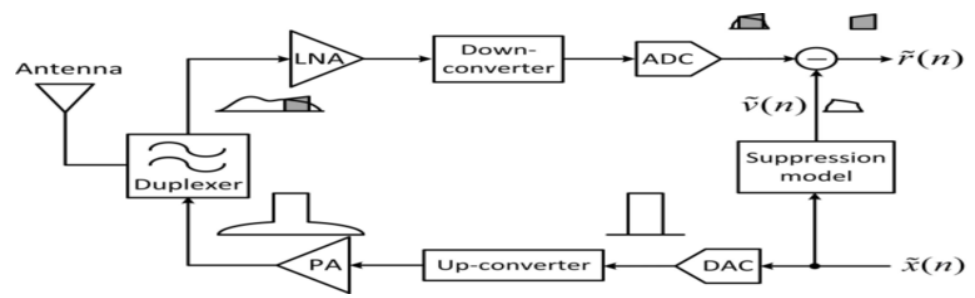

Figure 1. TX leakage at the receiver in FDD transceivers.

This circuit is embedded with low-noise trans-conductance amplifier (LNTA). Finally noise and distortion are minimized using the proposed circuit design model[6-7]. Andreas Gebhard et al.,(2019) proposed an algorithm using nonlinear type Recursive Least Square based Adaptive filter and its robust version cancelled the interference of the intermodulation distortion using second order. Here leakage of transmitter signal suppressed using stopband of frequency selective duplex[8]. H. Khatri et al.,(2010) and A. Cicalini et al.,(2006) proposed TX leakage filtering active cancellation techniques[9-11]. Lederer and Huemer (2011), Frotzscher and Fettweis (2008), and Kahrizi et al.,(2008) discussed interference cancellation algorithms using adaptive based LMS and measured error to suppress noise through stopband of the frequency flat duplex[12-13].

\section{Proposed work}

\subsection{OFDM Model}

The block diagram of communication wireless systems to transmit and receive data using more number of components required. Digital modulation technique is one of the important modules. This modulation allowed digitized data to be transmitted to the channel of the radio frequency. For without a break in continuity from this communication maintained using high transmission rate. MODEM provided fast and lossless transmission rate of data efficiently. Figure1 showed block diagram of proposed system. The required spectrum based on input data stream and modulation technique chose using OFDM system. Each carrier transmitted the assigned data. Using modulation techniques are required amplitude and phase of the carrier(i.e., BPSK, QPSK, or QAM). The required spectrum is then converted from frequency to time domain signal using an Inverse Fast Fourier Transform.

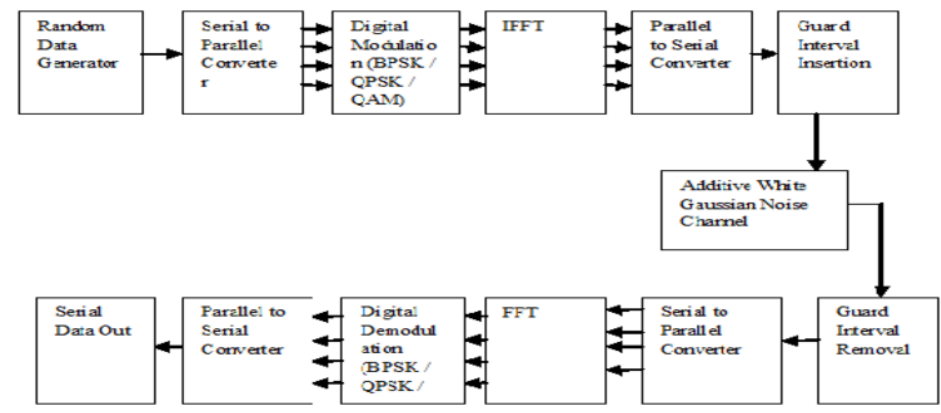

Figure 2. The proposed model used for simulation 


\subsection{Modulation Techniques}

In Day by day changing Communication System faces a problem of provided quality of services high with transmission errors are low. To overcome this problem using OFDM technology provided. BPSK and QPSK modulation techniques are used interleaving function. It is reduced the rate of transmission bit error. The transmission error rate of AWGN channel using QPSK and BPSK modulation have less bit error rate and also throughput of the signal strength is increased. Power consumption is consumed low power in this system.QAM is combination phase and amplitude. In higher-order constellation, it transmitted more number of Transmitted bits. The mean energy of the constellation are remained same. The points are closed together and it provided susceptible to noise and interference are high. In this three techniques we calculated error rate, increased throughput and minimize the power consumption also.

\section{Results and Discussion}

The data signal follows BPSK based on OFDM with 32 sub carriers, a fast fourier transform of size 64, 48 for data and 4 for pilots. The BER is computed on bits of the data and through an AWGN channel with various SNR. We considered that the noise plus signal is caused by RF Leakage and that signal has transmitted using same technique modulation parameters. The bit error rate is calculated using comparison of the input data stream from a transmitter to the input data stream from a receiver. SNR is done by the difference of signal and noise power. The results of the BER for various iteration of the same channel SNR. It is shown that the transmitter Leakage outcome performance and reduced consequence of this interference. Simulation of OFDM based QPSK was same setup function of BPSK based OFDM model. The only difference is that instead of BPSK modem replaced by the QPSK modem functions. Simulation of OFDM with 16-QAM modulation technique is combination of PSK and ASK. As usual BPSK technique, we calculated the parameters values, the same way QPSK and 16QAM same parameters values are calculated and mentioned below tables. The input data and channel model are same.
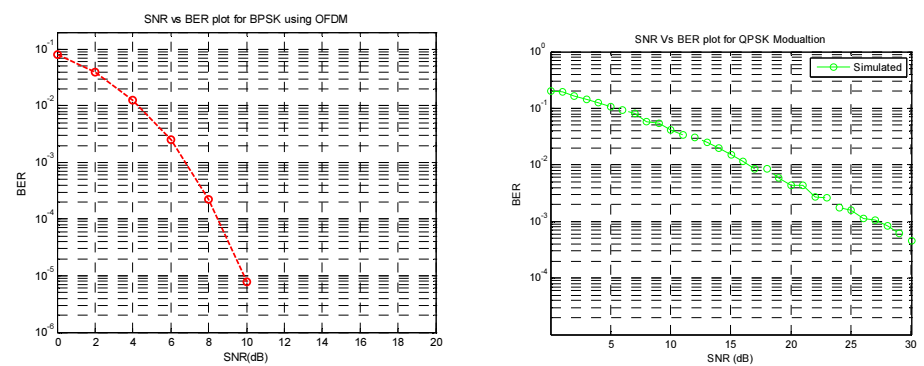

Figure 3. BER versus SNR curve for OFDM with BPSK and QPSK

From the graphical results (Figure 3), SNR values in $\mathrm{dB}$ are changed depending upon time in the Additive White Gaussian Noise channel. SNR value is calculated and their corresponding probability of error is also measured and tabulated. As we increased the SNR value, bit error rate reduced. 


\section{Performance Comparison}

Comparison of the various parameters values are mentioned in the below table using OFDM system modulation technique. From the results, it was observed that the BPSK allowed very low error transmission rate. Because it is used only two bits. In QPSK modulation, the throughput of the signal strength is increased compared to others. QAM is more susceptible to noise so they have a higher BER and compare to QPSK. So Signal to noise ratio is increased high in QPSK modulation compared to other techniques.

TABLE 1. Various parameter performance for OFDM model

\begin{tabular}{llllll}
\hline S.No & Modulation & SNR(dB) & BER & MSE & PSD \\
\hline 1. & QPSK & 33 & 0.2102 & 2.50 & 1.79 \\
2. & BPSK & 11 & 0.0802 & 1.73 & 1.60 \\
3. & 16QAM & 20 & 0.5016 & 2.57 & 1.34 \\
\hline
\end{tabular}

The various parameters are mentioned in table and also plotted graphical form of different techniques(Table 1, Figure 4). From the table shown QPSK is suppressed leakage of improving signals strength. In receiver side we got high throughput that meant transmission rate error was cancelled. Power consumption of this system value is 1.79 and Signal power noise ratio value is 33 .

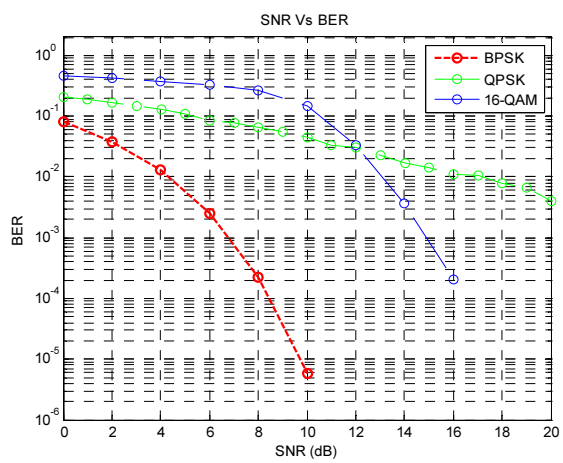

Figure 4. Comparison of Graph of BPSK, QPSK and 16-QAM.

\section{Conclusion}

Modulation and demodulation function is an important function in any communication system. It is decided the quality of the entire communication system. The proposed concept reduced the power consumption in the RF system and also increased throughput of the signal during reduced parity bit errors. OFDM was performed for different technique of digital modulation namely BPSK, QPSK and 16-QAM using FFT transform technique. From the results, BPSK allowed the Bit Error Rate improved in a noise channel data transmission capacity was maximized. Using QPSK allowed 
high transmission throughput. Therefore OFDM based QPSK required large transmission power. In this results, using OFDM with QPSK was more benifit for small distance of the link transmission. Otherwise OFDM based BPSK preferred. QAM is more susceptible to noise so they have a higher BER and compare to QPSK. So Signal to noise ratio is increased high in QPSK modulation compared to other techniques. Some more parameters also tabulated to analyse performance of three modulation techniques to minimize noise and increase SNR values.

\section{References}

[1] Cicalini et.al., An integrated LMS adaptive filter of TX leakage for CDMA receiver front ends, IEEE J. Solid-State Circuits, vol. 41, no. 5, pp. 1171-1182, May 2006.

[2] Frotzscher and G. Fettweis, A stochastic gradient LMS algorithm for digital compensation of Tx leakage in zero-IF receivers, in Proc. Veh. Technol. Conf. (VTC Spring), pp. 1067-1071, May 2008.

[3] Khatri.H, Gudem.P, and Larson.L, An active transmitter leakage suppression technique for CMOS SAW-less CDMA receivers, Solid- State Circuits, IEEE J. of, vol. 45, no. 8, pp. 1590-1601, 2010.

[4] Lederer and M. Huemer, LMS based digital cancellation of second order TX intermodulation products in homodyne receivers, in Proc. IEEE Radio Wireless Symp. (RWS), pp. 207-210, Jan. 2011.

[5] Frotzscher, G. Fettweis, Digital compensation of transmitter leakage in FDD zero-IF receivers, Trans. Emerging Telecommun. Tech, vol. 23, no. 2, pp. 105-120, March 2012.

[6] Lauri Anttila, Mikko Valkama Digital Suppression of Power Amplifier Spurious Emissions at Receiver Band in FDD Transceivers, IEEE Signal Processing Letters, Vol. 21, No. 1, January 2014.

[7] Jin Zhou et al., Low-Noise Active Cancellation of Transmitter Leakage and Transmitter Noise in Broadband Wireless Receivers for FDD/ Co-Existence, IEEE Journal Of Solid-state Circuits, Vol. 49, No. 12, December, 2014.

[8] Chao Yu, Wenhui Cao et al., Digital Compensation for Transmitter Leakage in Non-Contiguous Carrier Aggregation Applications with FPGA Implementation.IEEE Transactions On Microwave Theory And Techniques, Vol. 63, No. 12, December 2015.

[9] Z. Fu, L. Anttila, M. Abdelaziz, M. Valkama, and A. M. Wyglinski.Frequency-selective digital predistortion for unwanted emission reduction. IEEE Trans. Commun., vol. 63, no. 1, pp. 254-267, Jan. 2015.

[10] Wenhui Cao et al..Digital suppression of transmitter leakage in FDD RF transceivers: Aliasing elimination and model selection. IEEE Transactions On Microwave Theory And Techniques, 2017.

[11] Daniele Montanari, Giacomo Pini et al.,.An FDD Wireless Diversity Receiver with Transmitter Leakage Cancellation in Transmit and Receive Bands. IEEE Journal Of Solid-state Circuits, Vol. 53, No. 7, July 2018.

[12] Satheesh Bojja Venkatakrishnan, Elias A. Alwan et al., Wideband RF Self-Interference Cancellation Circuit for Phased Array Simultaneous Transmit and Receive Systems. IEEE Journal, Vol 6, 2018.

[13] Andreas Gebhard, Christian Motz et al..A Robust RLS Type Adaptive Filter for Second-OrderIntermodulation Distortion Cancellation in FDD LTE and 5G Direct Conversion Transceivers.IEEE Transactions On Microwave Theory And Techniques, Vol. 67, No. 5, May 2019.

[14] Kanchana devi.A, Bhuvaneshwari.B. A Study on Massive MIMO 5G Challenges. Advances in Parallel Computing Journal [IOS-Press], Scopus, DOI: 10.3233/APC200179.

[15] Nivetha.S.B,Bhuvaneswari.B .Investigation of Meandered Antenna for WLAN Application. Advances in Parallel Computing Journal [IOS-Press], Scopus, DOI: 10.3233/APC200167. 